4) Die einzelnen Strichcorrectionen werden völlig unabhängig von einander bestimmt. Dieser Umstand bretet die Möglichkeit einer sehr werthvollen Controle der ganzen Arbeit. Es seien $l, l^{\prime}, l^{\prime \prime}$ drei auf einander folgende $l$-Strecken auf der Mire, $n, n^{\prime}, n^{\prime \prime}$ die correspondirenden Werthe von $n$, ferner seien $u_{1} u_{2} u_{3} u_{4}$ - $u_{1}^{\prime} u_{2}^{\prime} u_{3}^{\prime} u_{4}^{\prime}-u_{1}^{\prime \prime} u_{2}^{\prime \prime} u_{3}^{\prime \prime} u_{4}^{\prime \prime}$ die benutzten Striche auf den Schieberskalen, derart, dass $u_{\alpha} u_{\alpha}^{\prime} u_{\alpha}^{\prime \prime}$ in derselben Gegend der betreffenden Skala liegen, endlich sei die Mirentheilung so eng gewählt, dass die Lage von $u_{\alpha}^{\prime}$ relativ gegen $u_{\alpha}$ und $u_{\alpha}^{\prime \prime}$ direct mittelst des Ablesemikrometers bestimmt werden kann und dass von $n$ bis $n^{\prime \prime}$ die Aenderung der Deviation als geradlinig anzusehen ist, dann liefert die mikrometrische Interpolation von $\delta n^{\prime}$ zwischen $\delta n$ und $\delta n^{\prime \prime}$ einen Werth von $\delta n^{\prime}$, der als eine völlig selbstständige Bestimmung von $\delta n^{\prime}$ zu betrachten ist und mit der directen Bestimmung aus $l$ vereinigt werden darf. Bei dem Leipziger Heliometer würde für den Winkelwerth von $l^{\prime}-l, l^{\prime \prime}-l^{\prime}$ der Betrag von rund $120^{\prime \prime}$ oder $160^{\prime \prime}$ eine passende Wahl sein.

5) Der erforderliche Arbeitsaufwand ist voraussichtlich merklich grösser, als bei einer directen Untersuchung der Schieberskalen, da die heliometrische Ablesung ausser den Skalenablesungen noch die Pointirung im Fernrohr erfordert, da ferner die Sicherheit dieser Pointirung ge- ringer ist, als die der Einstellung der Skalenstriche im Mikroskop, wenn sie auch für die Mirenstriche merklich grösser sein wird, als für Sterne. Dieser Nachtheil dürfte jedoch durch die Vortheile, namentlich aber durch den in 4) genannten Umstand aufgewogen werden.

6) In den Entwicklungen war unter $N$ immer das Maximalintervall $z u$ verstehen, welches bei dem in Rede stehenden Heliometer direct gemessen werden kann, d. h. im Winkel etwa $2^{\circ}$. Diese Festsetzung ist gerechtfertigt, so lange für die grossen Distanzen die Qualität der Bilder nicht wesentlich schlechter ist, als in der Mitte. Trifft diese Voraussetzung nicht $\mathrm{zu}$, so hat die Verwendung des Instruments als "Mikrometer c für die grossen Distanzen keinen $Z$ weck, und es ist dann auch überflissig, die Theilungsfehler zu untersuchen. In diesem Falle wird man also für $N$ denjenigen Grenzwerth setzen, bis zu welchem hin die heliometrische Messung andern Methoden noch überlegen oder ebenbürtig ist. Uebrigens dürfte, wenn bei grossen Distanzen die Bilder mangelhaft sind, dieses Hinderniss genauer Messung sich sehr wohl durch die von Prof. Abbe ursprünglich aus anderen Gründen vorgeschlagene Blende mit festem Ausschnitt allemal dann erheblich vermindern lassen, wenn es auf einen Lichtverlust nicht ankommt, denn der Mangel rührt bei den modernen Heliometern wesentlich von dem Verhalten der Medien Ocular-Auge her.

Leipzig 1885 Juni 22.

\title{
Entdeckung eines neuen Cometen durch Herrn Barnard.
}

Am I o. Juli Nachmittags erhielt die Centralstelle folgendes Telegramm aus Boston:

-Urbane Barnard ukase July cartouch keen trarbuncled treat constablery rascal smallness unhappy cordage. Harvard Obs.*

Nach einer leicht ersichtlichen Correctur, carbuncled für trarbuncled, lautet das Telegramm:

The announcement has been received by Harvárd College Observatory from Mr. Lewis Swift, Director of »the Warner Observatory, of the discovery of a comet by Barnard July 7 .

July 9.7204 Greenw. M. T. RA. $=259^{\circ} 27^{\prime} 6^{\prime \prime}$ NPD. $=96^{\circ} 1^{\prime} 8^{\prime \prime}$. Daily motion in RA.: - $30^{\circ}$, in NPD.: +35'. * The physical appearance of the comet is as follows: Circular, diameter $I^{\prime}$, brightness $\mathbf{I}^{\mathrm{m}}$ or fainter, some * central condensation, no tail.

Harvard Observatory.

Der Comet wurde von Dr. Lamp am 10. und 1 r. Juli Abends im hiesigen Refractor gesehen, eine Ortsbestimmung konnte jedoch wegen der Dämmerung und nicht günstiger Luft nicht erlangt werden.

Die folgenden Beobachtungen gingen am I 2. resp. I3. Juli bei der Centralstelle telegraphisch ein :

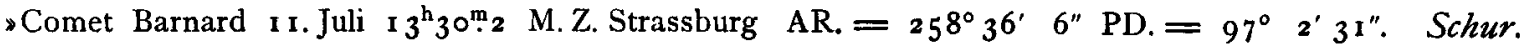

$$
\begin{aligned}
& \text { 12. Juli } 940.0 \text { M.Z. Palermo }=25^{8} \mathbf{1 3}_{32}=973^{1} 3^{8} \text {. Cacciatore.* }
\end{aligned}
$$

Die beiden Telegramme aus Boston und Strassburg wurden an die Mitglieder der Centralstelle sofort nach ihrem Eintreffen weiter befördert.

Kiel 1885 Juli 13. 